\title{
Evaluación de la competencia transversal "Análisis y resolución de problemas" (CT3) en la titulación de GADE de la Facultad de ADE de la Universitat Politècnica de València
}

\author{
Cortés López, Juan Carlos ${ }^{\mathrm{b}}$; Mateos Ronco, Alicia ${ }^{\mathrm{a}}$; Santamaría Navarro, Cristina ${ }^{\mathrm{b}}$; \\ Villanueva Micó, Rafael ${ }^{b}$ \\ aDepartamento de Economía Y Ciencias Sociales, Universitat Politècnica de València; almaron@esp.upv.es; \\ bDepartamento de Matemática Aplicada, Universitat Politècnica de València; jccortes@imm.upv.es, \\ crisanna@imm.upv.es, rjvillan@imm.upv.es
}

\begin{abstract}
This paper describes the development and the conclusion of the meeting the professors of the Faculty of Business Administration in charge of the "point of control" subjects of the soft skill "Problem Analysis and Solving" (CT3) in the first and second level, held May $6^{\text {th }}, 2019$. The professors share their experience in implementing assessment methods in every subject following the guidelines of the general project of the UPV.

The main conclusion is that the professor feels alone and has to find imaginative methods to assess the soft skill in a reliable way. Also, extant multiplicity of assessments of the skill contribute to certain confusion in the final consequence of the assessment in each subject, and hence a simplification is proposed.
\end{abstract}

Keywords: soft skills, assessment, business administration degree, problem analysis and solving.

\section{Resumen}

En este articulo se describe el desarrollo y las conclusiones alcanzadas en una reunión celebrada el 6 de mayo de 2019 de los profesores de la Facultad de Administración y Dirección de Empresas (FADE) responsables de asignaturas que son punto de control de la competencia transversal "Análisis y resolución de problemas" (CT3) en primer y segundo nivel. Los profesores compartieron su experiencia en la implementación de las directrices del proyecto de competencias transversales de la UPV y la correspondiente evaluación en cada una de las asignaturas.

Se concluye que el profesor se encuentra solo y tiene que ingeniárselas para realizar una evaluación de la CT3 fiable y coherente. Además, se propone una reducción en las CTs asignadas a cada asignatura.

Palabras clave: competencias transversales, evaluación, grado en ADE, análisis y resolución de problemas. 


\section{Introducción}

En este trabajo vamos a plasmar la discusión, puntos de vista y conclusiones alcanzadas en una reunión, el pasado 6 de mayo de 2019, donde los profesores de la FADE responsables de las asignaturas con la competencia transversal 3 (CT3) compartieron sus experiencias en la puesta en marcha del proyecto de competencias transversales en cada una de sus asignaturas.

Para esto, dividiremos el artículo como sigue. En la sección 2 haremos una descripción general del proyecto de competencias transversales de la UPV para situarnos en contexto. En la sección 3 describiremos en qué consiste la CT3. En la sección 4 enumeraremos las asignaturas que tienen asignada la CT3 y qué tipo de punto de control son. Asimismo, describiremos, de manera agrupada, las diversas técnicas que se utilizan para evaluar la CT3. Finalmente, en la sección 5 resumiremos las reflexiones más relevantes que se realizaron así como las conclusiones más importantes.

\section{El proyecto de competencias transversales de la UPV}

De acuerdo con la filosofía del Espacio Europeo de Educación Superior (EEES) de formar a los alumnos en competencias, y entendiendo que éstas no deben ceñirse exclusivamente al ámbito de la técnica en cada titulación, la Universitat Politècnica de Valéncia (UPV) viene desarrollando un Proyecto de implantación de Competencias Transversales en sus titulaciones.

Podemos definir las competencias transversales como aquellas habilidades relacionadas con el desarrollo personal, que no dependen de un ámbito temático o disciplinario específico sino que aparecen en todos los dominios de la actuación profesional y académica (González y Wagenaar, 2003).

Este proyecto tiene como objetivo principal acreditar las competencias transversales de la UPV a los estudiantes egresados en cualquiera de los títulos oficiales impartidos en la UPV.

Con la puesta en marcha de este proyecto, podemos afirmar que la UPV va a estar en situación de:

- Proporcionar a sus egresados un valor añadido que puede diferenciarlos de otros egresados y, en consecuencia, hacer más atractivos los estudios ofertados frente a ofertas similares de otras universidades.

- Poner en valor la capacitación de nuestros egresados de cara a los empleadores.

- Explicitar la adquisición de las competencias de cara a acreditaciones internacionales.

La UPV tiene definidas tres vías de evaluación para las competencias transversales:

1. Durante el proceso formativo: a través de asignaturas que son punto de control. 
2. Al finalizar los estudios: a través del Trabajo Fin de Grado y del Trabajo Fin de Máster.

3. A través de actividades extracurriculares que evidencien el nivel alcanzado en cualquiera de las competencias transversales.

A continuación, se muestra la relación de competencias transversales, así como el concepto que valora:

CT1 Comprensión e integración. Demostrar la comprensión e integración del conocimiento tanto de la propia especialización como en otros contextos más amplios.

CT2 Aplicación pensamiento práctico. Aplicar los conocimientos a la práctica, atendiendo a la información disponible, y estableciendo el proceso a seguir para alcanzar los objetivos con eficacia y eficiencia.

CT3 Análisis y resolución de problemas. Analizar y resolver problemas de forma efectiva, identificando y definiendo los elementos significativos que lo constituyen.

CT4 Innovación, creatividad y emprendimiento. Innovar para responder satisfactoriamente y de forma original a las necesidades y demandas personales, organizativas y sociales con una actitud emprendedora.

CT5 Diseño y proyecto. Diseñar, dirigir y evaluar una idea de manera eficaz hasta concretarla en un proyecto.

CT6 Trabajo en equipo y liderazgo. Trabajar y liderar equipos de forma efectiva para la consecución de objetivos comunes, contribuyendo al desarrollo personal y profesional de los mismos.

CT7 Responsabilidad ética, medioambiental y profesional. Actuar con responsabilidad ética y profesional ante uno mismo y los demás.

CT8 Comunicación efectiva. Comunicarse de manera efectiva, tanto de forma oral como escrita, utilizando adecuadamente los recursos necesarios y adaptándose a las características de la situación y de la audiencia.

CT9 Pensamiento crítico. Desarrollar un pensamiento crítico interesándose por los fundamentos en los que se asientan las ideas, acciones y juicios, tanto propios como ajenos.

CT10 Conocimiento de los problemas contemporáneos. Conocimiento de los problemas contemporáneos.

CT11 Aprendizaje permanente. Utilizar el aprendizaje de manera estratégica, autónoma y flexible, a lo largo de toda la vida, en función del objetivo perseguido.

CT12 Planificación y gestión del tiempo. Planificar adecuadamente el tiempo disponible y programar las actividades necesarias para alcanzar los objetivos, tanto académico-profesionales como personales. 
CT13 Instrumental específica. Capacidad para utilizar las técnicas, las habilidades y las herramientas actualizadas necesarias.

Este proyecto ha establecido tres niveles de dominio para cada competencia transversal: el $1^{\text {er }}$ nivel en asignaturas de $1^{\circ}$ y $2^{\circ}$ cursos de grado, el $2^{\circ}$ nivel para asignaturas de $3^{\circ}$ y $4^{\mathrm{o}}$ curso del grado y el $3^{\text {er }}$ nivel para asignaturas de máster. En los grados, las competencias transversales han sido asignadas a asignaturas concretas de los planes de estudio.

Entre los diferentes instrumentos de evaluación que podemos utilizar para evaluar el desarrollo de las competencias transversales, las rúbricas se nos presentan como la alternativa más recomendada por los especialistas en el tema. Una rúbrica (también denominada matriz de valoración) es un recurso para la evaluación y calificación del aprendizaje, de los conocimientos o del desempeño de los estudiantes en una actividad concreta (o en un módulo, bloque o materia) y que establece criterios o indicadores y una escala de valoración para cada uno de ellos. Permite clarificar lo que se espera del trabajo del alumno, valorar su ejecución y facilitar la realimentación. La calificación final de las Competencias Transversales se basa en un sistema cualitativo alfabético de 4 valores en el que:

- A: la competencia se supera excelentemente,

- B: La competencia se alcanza completamente,

- C: la competencia se alcanza parcialmente,

- D: la competencia no se alcanza, es decir, el criterio no logra el nivel mínimo.

Ese escalado debe reflejar el nivel del estudiante en dicha competencia.

Puede encontrarse amplia información sobre el Proyecto de Competencias Transversales de la UPV en la página web http://www.upv.es/contenidos/COMPTRAN/.

\section{CT3: Análisis y resolución de problemas}

Como hemos visto anteriormente, la CT3 corresponde al análisis y resolución de problemas, y tiene como objetivo evaluar cómo el alumno analiza y resuelve problemas de forma efectiva, identificando y definiendo los elementos significativos que lo constituyen.

Los problemas son situaciones nuevas que requieren que los individuos respondan con comportamientos nuevos. Resolver un problema implica realizar tareas que demandan procesos de razonamiento más o menos complejo y, en muchas ocasiones, no simplemente una acción asociativa y rutinaria. El objetivo de esta competencia es, pues, que el alumno sea capaz de aplicar procedimientos estructurados para resolver problemas, promoviendo así su capacidad de aprender, comprender y aplicar conocimientos de forma autónoma (Universitat Politècnica de Valéncia, 2019). 
El proyecto institucional de la UPV propone las siguientes metodologías e instrumentos de evaluación recomendados:

- Actividades: Actividades grupales, Estudio de casos, Exposiciones orales, Lecturas, Prácticas laboratorio, Preguntas, Problemas, Proyectos y Seminario.

- Evaluación: Autoevaluación, Caso, Evaluación entre iguales, Examen/Exposición oral, Mapas conceptuales, Observación, One minute paper, Prueba escrita de respuesta abierta, Pruebas objetivas, Redacción de informes, Rúbricas y Sondeo.

\section{Evaluación de la competencia CT3 "Análisis y resolución de problemas" en el GADE-UPV}

Las asignaturas reflejadas en la tabla 1 son los puntos de control de la competencia transversal "análisis y resolución de problemas" en el grado en ADE.

Tabla 1. Asignaturas punto de control de la CT3 "Análisis y resolución de problemas" en el GADE, nivel y curso de impartición

\begin{tabular}{ccc}
\hline Nivel & Curso & Asignatura \\
\hline 1 & 1 & Introducción a la Estadística \\
\hline 1 & 1 & Modelos Matemáticos para ADE I \\
\hline 1 & 1 & Modelos Matemáticos para ADE II \\
\hline 1 & 1 & Microeconomía I \\
\hline 1 & 1 & Contabilidad Financiera y de \\
\hline 1 & 2 & Sociedades \\
\hline 2 & 2 & Análisis y Consolidación Contable II \\
\hline 2 & 3 & Investigación Operativa \\
\hline 2 & 3 & Métodos Cuantitativos para la \\
& 3 & La Comunicación en la Empresa \\
\hline 2 & 4 & Análisis del Riesgo Financiero \\
\hline 2 & 4 &
\end{tabular}

Fuente: Elaboración propia.

Los profesores responsables de estas asignaturas fueron convocados a una reunión de coordinación para poner en común reflexiones, inconvenientes y metodologías utilizadas en la evaluación de la competencia en sus respectivas materias de la titulación. La 
reunión, celebrada el 6 de mayo de 2019 en la Facultad de ADE, contó con 10 asistentes y en ella se abordaron los siguientes espectos: metodologías aplicadas en cada asignatura para la evaluación de la competencia; ventajas e inconvenientes detectados en la aplicación de estas metodologías. La reunión sirvió para que aflorasen las limitaciones y dificultades encontradas, así como posibles soluciones y/o recomendaciones para los órganos competentes.

Sobre las metodologías de evaluación de la CT3 que se utilizan en las asignaturas de la tabla 1, las reflexiones realizadas se podrían resumir como siguen:

1. En la mayoría de los casos, el sistema de evaluación de la CT3 está incluido en el propio de la asignatura. Los alumnos deben responder, en los actos de evaluación, a situaciones que requieren el análisis y resolución de problemas propios de la materia en contextos económicos. Por ello no es necesaria la realización de pruebas más concretas. En consecuencia, la adquisición de la CT3 está muy relacionada con superar la asignatura en la que se evalúa.

2. Se utilizan pruebas escritas cronometradas, efectuadas bajo control del profesorado, en las que el alumno construye su respuesta; y/o pruebas de tipo test cronometradas, bajo control de los profesores, en las que el alumno no elabora la respuesta, sólo ha de señalarla o completarla con elementos muy precisos. Estas pruebas se realizan tanto en aula (pruebas de evaluación) como en laboratorio informático (prácticas).

3. En algunos casos, las pruebas de evaluación de la asignatura recogen algún apartado (pregunta o cuestión) específico para la evaluación de la CT3.

4. Los alumnos analizan la veracidad de noticias aparecidas en los medios de comunicación escritos. Para ello, deben escoger las variables que deben tenerse en cuenta, buscarlas en la memoria anual de la empresa (si es el caso), proponer un modelo adecuado al problema que se analiza, explicar sus parámetros y establecer las restricciones que deben plantearse sobre los mismos para contrastar la afirmación realizada en la noticia en cuestión.

5. Los aspectos que se evalúan en general en las asignaturas y, por añadidura, en la CT3 son: correcta aplicación de las técnicas de resolución; interpretación/análisis de resultados; exposición ordenada de las ideas; saber expresarse por escrito con corrección.

6. La evaluación de la CT3 se realiza transformando la nota obtenida en la escala de los niveles de la competencia. La nota podrá ser individual o conjunta para varios alumnos, en caso de que se evalúe mediante trabajos en grupo (por ejemplo, evaluación de prácticas). En este último caso, la observación de la dinámica de trabajo del grupo puede matizar la nota de cada miembro. 


\section{Reflexiones y conclusiones}

Las ventajas e inconvenientes observados durante este tipo de experiencia en el proyecto de las competencias transversales, son:

- Necesidad de medios adecuados: los tamaños de grupo apropiados (20-25 alumnos) facilitan la evaluación individualizada de la CT3, de forma además asequible en tiempo para el profesorado. Sin embargo, el elevado número de alumnos, particularmente en los grupos de las asignaturas de los primeros cursos del Grado, dificulta e incluso impide la aplicación de sistemas específicos de evaluación de la CT3, adicionales a los propios de la materia.

- La correlación total existente entre la nota final de la asignatura y la evaluación de la CT3 podría plantear el inconveniente de que puede tratarse de una información duplicada en la evaluación del alumno, si bien, también asegura una evaluación de la CT3 de una forma fiable y adecuada, que puede verse distraida por la evaluación de otras CTs de la asignatura.

- Aunque no es el caso concreto de la CT3, la asignación de ciertas competencias de forma "forzada" a las asignaturas plantea inconvenientes al profesorado, que no acaba de tener clara su vinculación con la materia que imparte $\mathrm{y}$, en consecuencia, encuentra dificultades para diseñar su sistema de evaluación.

- La capacitación en CT requiere de unas condiciones estructurales: un diseño general de todo el Grado, para asignar debidamente las competencias de modo natural a las asignaturas que por su naturaleza pueden incorporarlas (la competencia no es sólo un saber (teoría), sino un saber hacer (teoría aplicada) y poder hacer (prácticas)); un plan de implantación, que garantice que se haga de forma escalonada y gradual en el tiempo; una cultura organizativa que alinee a los profesores.

- Existen opiniones diversas en relación a la necesidad de una capacitación explícita del profesorado en competencias. El profesorado tiene sólidos conocimientos de la materia que imparte, pero no parece realista presuponer que las tiene también sobre el modo de formar a sus alumnos en la CT que tiene que evaluar. Se exponen opiniones a favor de esta capacitación para el profesorado, y también en contra, entendiendo que no es el profesorado el que debe asumir este rol.

- Lo anterior ha generado intercambio de opiniones sobre la forma en que el proyecto institucional de Competencias Transversales se está plasmando en la realidad de nuestras aulas y asignaturas. En este sentido, algunos profesores apuntan que no parece suficientemente explorado un aspecto crucial como es la formación del alumnado en dichas competencias, por lo que plantear una evaluación final de las mismas sin esta formación previa adolece de cierta incoherencia.

- Asimismo, creemos que sería más práctico que cada asignatura solo fuera punto de control de la competencia transversal más relacionada. Entendemos que permitiría realizar una evaluación de la competencia más fiable y ajustada. El 
hecho de que las mismas competencias se evalúen en varias asignaturas permitiría aplicar esta sugerencia, así como se reducirían las confusiones y redundancias que se pueden producir al asignar distintas evaluaciones de una misma competencia desde distintas asignaturas.

- Por otra parte, las asignaturas que tienen una segunda parte como Modelos Matemáticos para ADE o Microeconomía, entre otras, sería interesante que las competencias sólo se evaluaran en la segunda parte de la asignatura, evitando efectos no deseados como que un alumno que supere la competencia en la asignatura inicial y la suspenda en la asignatura de continuación. Este tipo de disfunciones, además, desconocemos cómo se resuelve finalmente y cómo aparece en el expediente del alumno.

\section{Referencias}

GONZALEZ, J.; WAGENAAR, R. (2003). Tuning Educational Structures in Europe. Informe Final. Bilbao: Universidad de Deusto. http://www.ub.edu/cubac/sites/default/files/tuning educational structures espanyol 0.p df. [Consulta: 26 de mayo de 2019].

UNIVERSITAT POLITÈCNICA DE VALÈNCIA. Competencias transversales. http://www.upv.es/contenidos/COMPTRAN/ [Consulta: 26 de mayo de 2019].

UNIVERSITAT POLITÈCNICA DE VALÈNCIA. Competencia Análisis y Resolución de Problemas. http://www.upv.es/contenidos/COMPTRAN/info/954723normalc.html. [Consulta: 26 de mayo de 2019]. 